\title{
Fatigue reliability assessment of welded bridge details using probabilistic fracture mechanics
}

\author{
John Leander \\ KTH Royal Institute of Technology, Stockholm, Sweden
}

Farshid Zamiri, Mohammad Al-Emrani

Chalmers University of Technology, Gothenburg, Sweden

Contact: john.leander@byv.kth.se

\begin{abstract}
The safe life method for the fatigue life prediction of steel bridges has for several cases been shown to render misleading results. To improve the accuracy, a probabilistic model based on linear elastic fracture mechanics (LEFM) is suggested. The model is used for a sensitivity analysis on the influence of different modelling options pertinent for a fatigue assessment of bridge details. The influence of material parameters and the crack growth threshold have been investigated. The analyses have been performed for a bridge detail frequently occurring in old steel bridges and a stress range spectrum based on measurements. The results show a significant difference in the estimated fatigue life depending on the modelling options. Furthermore, the omission sensitivity factors have been estimated for the probabilistic model showing the influence of the uncertainties of the variables and which of them that needs to be determined with care.
\end{abstract}

Keywords: Life prediction; Reliability; Linear elastic fracture mechanics (LEFM).

\section{Introduction}

The aging infrastructure in developed countries is an impending economic burden. Many structures have reached their expected service life which forces decisions on remedial actions. To support these decisions and avoid costs and resource depletion due to ill-judged actions, accurate service life predictions are required.

For steel bridges, the service life is typically limited by fatigue. The safe life method is the prevailing philosophy for assessment where the remaining fatigue life is calculated using load models, resistance parameters and partial safety factors specified in design codes for the design of new bridges. All the input parameters and the model itself are afflicted with uncertainties. In particular the load history is rarely known in detail for existing bridges which makes the service life prediction highly unreliable. Examples from practice have shown a theoretically exhausted fatigue life while no signs of damage have been found during inspection [1]. In other examples, unanticipated fatigue cracking has been reported for various bridge details due to secondary effects, sometimes already after few years in service [2].

The damage tolerant method is an alternative approach which allows an assessment of the service life in the presence of a fatigue crack, assumed or existing. The load history can with this method be disregarded. The previous accumulated damage is represented by an initial 
crack depth. The mathematical tool for a fatigue assessment following the damage tolerant method is linear elastic fracture mechanics (LEFM) [3].

LEFM provides a better representation of the fatigue deterioration process than the safe life method. The damage is represented by a physical entity - the crack depth, and the nonlinear relation between number of cycles and damage is captured. Therefore, a damage tolerant method is expected to provide more accurate estimations of the remaining fatigue life. There is, however, no deterministic design format based on LEFM available in today's standards for bridges. To investigate the influence of different modelling options and uncertainties on the estimated fatigue life, a probabilistic model is suggested in the current paper and used for sensitivity analyses. The model enables considerations of the scatter in the input parameters, model uncertainties, and an assessment against a stipulated reliability level.

The purpose of the current paper is to elucidate some of the uncertainties surrounding an assessment based on LEFM. The objective is to compare different material models for the fatigue crack growth rate which needs to be established for a future development of a deterministic format.

A long term aim is to develop a method for fatigue life predictions more accurate than the safe life method to support decisions on remedial actions for existing bridges.

\section{Probabilistic model}

Stable growth of a fatigue crack can be described by the well-known Paris law [4]

$$
\frac{d a}{d N}=A K_{\mathrm{r}}^{n}
$$

where $a$ is the crack depth, $N$ is the number of cycles, and $A$ and $n$ are material dependent constants. The stress intensity factor range $K_{\mathrm{r}}$ in Eq. (1) is dependent on the stress range $S_{\mathrm{r}}$ and the crack depth $a$ as

$$
K_{\mathrm{r}}=S_{\mathrm{r}} \sqrt{\pi a} Y(a) M_{\mathrm{k}}(a)
$$

where $Y(a)$ is a geometry correction factor for the unwelded component, and $M_{\mathrm{k}}(a)$ is a stress magnification factor for the weld geometry [5]. The number of cycles required to propagate a crack from an initial crack depth of $a_{0}$ to a crack depth of $a_{\mathrm{c}}$ is obtained by integrating the crack growth rate

$$
N_{\mathrm{c}}=\int_{a_{0}}^{a_{\mathrm{c}}}\left(\frac{d a}{d N}\right)^{-1} d a
$$

A general design equation for fatigue assessment can be formulated as

$$
M(t)=N_{\mathrm{c}}-N(t)
$$

where $M(t)$ is the time variant safety margin, $N_{\mathrm{c}}$ is the number of cycles to reach a critical crack depth of $a_{\mathrm{c}}$ as described by Eq. (3), and $N(t)$ is the number of cycles reached at time $t$. A safety margin equal or less than zero indicates an exhausted fatigue life.

\subsection{Limit state equation}

For the reliability analyses, a limit state equation based on Eq. (4) was formulated as

$$
g(\mathbf{x}, t)=N_{\mathrm{c}}(\mathbf{x})-N(t)
$$

where $N_{\mathrm{c}}(\mathbf{x})$ is determined by Eq. (3) for the stochastic variables contained in the vector $\mathbf{x}$. A state of failure is defined for $g \leq 0$ and the probability of failure is defined as $P_{\mathrm{f}}=P(g \leq 0)$ [6]. The reliability index $\beta$ is related to the probability of failure as

$$
\beta=-\Phi^{-1}\left(P_{\mathrm{f}}\right)
$$

where $\Phi^{-1}()$ is the inverse of the standardized normal distribution function. 


\subsection{Crack growth rate}

For the analyses presented in the current paper, the linear Paris law was expanded to a bi-linear crack growth rate suggested in BS 7910 [7]. It is described by a conditional differential equation as

$$
\frac{d a}{d N}= \begin{cases}0, & \left(C_{\mathrm{S}} K_{\mathrm{r}}\right)<K_{\mathrm{th}} \\ A_{\mathrm{a}}\left(C_{\mathrm{S}} K_{\mathrm{r}}\right)^{n_{\mathrm{a}}}, & K_{\mathrm{th}} \leq\left(C_{\mathrm{S}} K_{\mathrm{r}}\right)<K_{\mathrm{ab}} \\ A_{\mathrm{b}}\left(C_{\mathrm{S}} K_{\mathrm{r}}\right)^{n_{\mathrm{b}}}, & \left(C_{\mathrm{S}} K_{\mathrm{r}}\right) \geq K_{\mathrm{ab}}\end{cases}
$$

where the transition point $K_{\mathrm{ab}}$ can be calculated as

$$
K_{\mathrm{ab}}=\left(\frac{A_{\mathrm{a}}}{A_{\mathrm{b}}}\right)^{1 /\left(n_{\mathrm{b}}-n_{\mathrm{a}}\right)}
$$

A threshold value $K_{\text {th }}$ is considered in Eq. (7), below which no crack growth occurs. The variable $C_{\mathrm{S}}$ in the same equation represents the uncertainty of the stress intensity factor range and should be split into three variables as [8]

$$
C_{\mathrm{S}}=C_{\mathrm{GLB}} C_{\mathrm{SCF}} C_{\mathrm{SIF}}
$$

where $C_{\mathrm{GLB}}, C_{\mathrm{SCF}}$ and $C_{\mathrm{SIF}}$ are the model uncertainties of the global stress model, the stress concentration, and the stress intensity factor, respectively. Recommended statistical distributions can be found in [8].

Bridges are subjected to variable amplitude loading. The response is typically presented as a stress range spectrum showing the number of cycles in each stress range. A spectrum based on measured strains from the Söderström Bridge, a railway bridge in Sweden, is shown in Fig. 1. The bridge and the monitoring campaign are presented in [1].

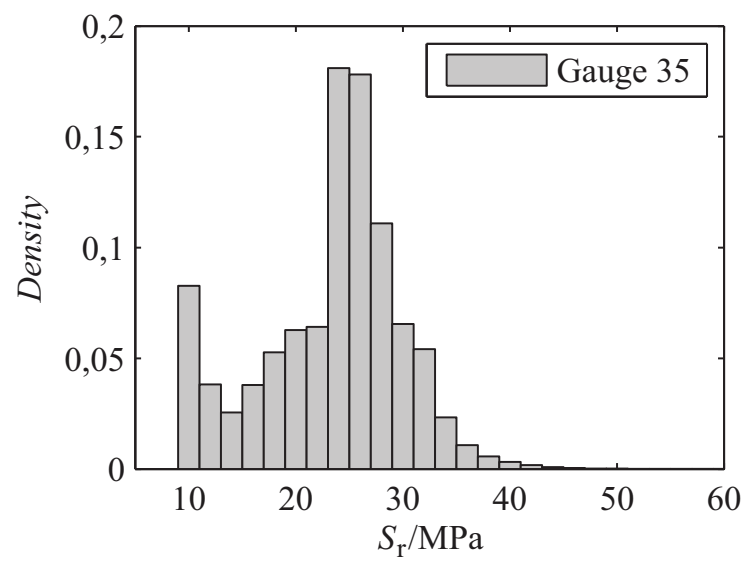

Figure 1. A stress range spectrum based on measured strains from the Söderström Bridge [1].

For variable amplitude loading, the integration in Eq. (3) has to be performed cycle by cycle or for blocks of cycles with the same stress range. Assuming that the stress variation is an ergodic process, the load sequence can be neglected and an expected crack growth rate can be used [8]

$$
\begin{aligned}
E\left[\frac{d a}{d N}\right]= & A_{\mathrm{a}} E\left[S_{\mathrm{r}}^{n_{\mathrm{a}}}\right]_{S_{\mathrm{th}}}^{S_{\mathrm{ab}}}\left(C_{\mathrm{S}} \sqrt{\pi a} Y(a) M_{\mathrm{k}}(a)\right)^{n_{\mathrm{a}}} \\
& +A_{\mathrm{b}} E\left[S_{\mathrm{r}}^{n_{\mathrm{b}}}\right]_{S_{\mathrm{ab}}}^{\infty}\left(C_{\mathrm{S}} \sqrt{\pi a} Y(a) M_{\mathrm{k}}(a)\right)^{n_{\mathrm{b}}}
\end{aligned}
$$

where $E[]$ denotes the expected value. The stress range limits $S_{\text {th }}$ and $S_{\text {ab }}$ corresponds to the values reaching $K_{\text {th }}$ and $K_{\mathrm{ab}}$, respectively. Using the expected crack growth rate reduces the computational effort significantly in comparison to a cycle by cycle integration.

\subsection{Reliability analysis}

The reliability analyses were performed using the first order reliability method (FORM). The stochastic variables were described by mean values, standard deviations and their statistical distribution functions. Non-normal distributed variables were considered using the so-called normal tail approximation [6]. The calculations were performed in Matlab [9] using a constrained nonlinear optimization algorithm. 


\subsection{Uncertainties}

The variables considered are listed in Table 1 together with their statistical distributions. These properties serve as the reference case in the sensitivity analyses. Most variables are assigned properties as suggested in the Probabilistic model code [8]. An exception is the model uncertainty related to the global stress. In this paper, the stresses considered were determined by measurements which imply a higher accuracy than obtained with a theoretical calculation. The uncertainty of the global stress was modelled as suggested in [10] with a lognormal distribution, a mean value of unity, and a coefficient of variation $(\mathrm{CoV})$ of $3 \%$. No stress concentration factor is considered why $C_{\mathrm{SCF}}$ in Eq. (9) is omitted.

The critical crack depth $a_{\mathrm{c}}$ was set to a deterministic value in the calculations. It was selected sufficiently large to render a crack growth rate close to infinite and, thereby, having a negligible influence on the estimated fatigue life.

Table 1. Stochastic variables for the reference case. $N \sim$ Normal, $L N \sim$ Lognormal,

$D E T \sim$ Deterministic. The values are valid for crack growth in $\mathrm{mm} /$ cycle and stress intensity in $\mathrm{N} / \mathrm{mm}^{3 / 2}$.

\begin{tabular}{cccc}
\hline Variable & Distribution & Mean & CoV \\
\hline$C_{\mathrm{GLB}}$ & $\mathrm{LN}$ & 1 & 0,03 \\
\hline$C_{\mathrm{SIF}}$ & $\mathrm{LN}$ & 1 & 0,07 \\
\hline$S_{\mathrm{r}}$ & $\mathrm{DET}$ & - & - \\
\hline$A_{\mathrm{a}}$ & $\mathrm{LN}$ & $4,80 \cdot 10^{-18}$ & 1,70 \\
\hline$A_{\mathrm{b}}$ & $\mathrm{LN}$ & $5,86 \cdot 10^{-13}$ & 0,60 \\
\hline$n_{\mathrm{a}}$ & $\mathrm{DET}$ & 5,10 & - \\
\hline$n_{\mathrm{b}}$ & $\mathrm{DET}$ & 2,88 & - \\
\hline$K_{\mathrm{th}}$ & $\mathrm{LN}$ & 140 & 0,40 \\
\hline$a_{0}$ & $\mathrm{LN}$ & 0,15 & 0,66 \\
\hline$a_{\mathrm{c}}$ & $\mathrm{DET}$ & $w / 2$ & - \\
\hline
\end{tabular}

\section{Sensitivity analysis}

Different modelling options for the material dependent properties of the crack growth rate have been investigated. It involves issues as the stress intensity threshold, linear or bi-linear crack growth rate, and bridge specific material parameters.

The calculations were performed for a specific detail from the Söderström Bridge shown in Fig. 2. It is a gusset plate welded to the flange of an Ishaped stringer beam. The purpose of the gusset plate is to attach the bracing to the stringer. As indicated in Fig. 2, a crack is assumed to initiate at the end of the butt weld between the gusset plate and the flange and propagate into the flange.

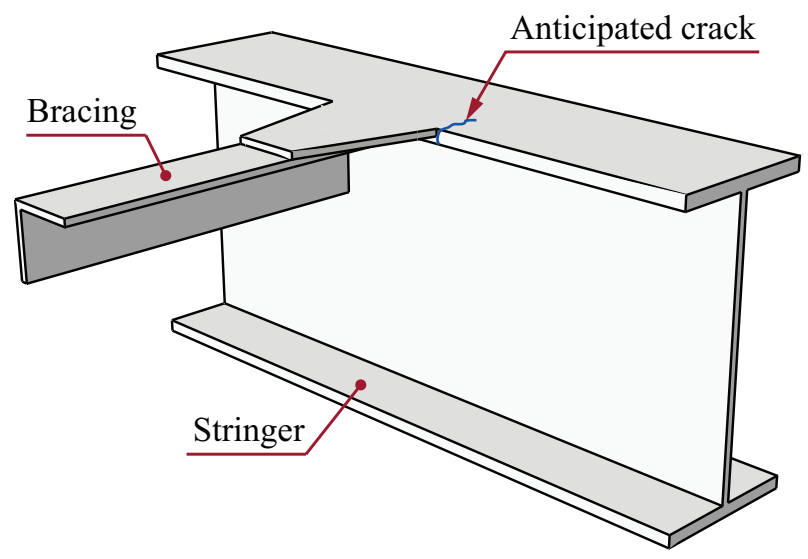

Figure 2. A gusset plate welded to the flange of a stringer beam. The anticipated crack is assumed to propagate into the top flange.

The geometry correction factor $Y(a)$ in Eq. (2) for the detail is taken as for a single edge notch specimen in tension (SENT) given in [11] as

$$
Y\left(\frac{a}{w}\right)=\sqrt{\frac{2 w}{\pi a} \tan \frac{\pi a}{2 w}} \frac{0,752+2,02\left(\frac{a}{w}\right)+0,37\left(1-\sin \frac{\pi a}{2 w}\right)^{3}}{\cos \frac{\pi a}{2 w}}
$$

where $a$ is the crack depth and $w$ is the plate width. The stress magnification factor, $M_{\mathrm{k}}(a)$, for this connection is given in [12] as 


$$
\begin{aligned}
M_{\mathrm{k}}\left(\frac{a}{w}\right)=b_{1} & +b_{2} \exp \left(b_{3} \frac{a}{w}\right) \\
& +b_{4} \exp \left(b_{5} \frac{a}{w}\right)
\end{aligned}
$$

where $b_{1}$ to $b_{5}$ are coefficients dependent on the dimensions of the connection. In the current study, the main plate was assigned dimensions $w=225 \mathrm{~mm}$ and $t=20 \mathrm{~mm}$, and the gusset plate the length and thickness values $200 \mathrm{~mm}$ and $10 \mathrm{~mm}$, respectively. These values represent a connection in the Söderström Bridge. The associated coefficients are $b_{1}=1,025 ; b_{2}=3,005$; $b_{3}=-858,4 ; b_{4}=1,220 ;$ and $b_{5}=-47,13$.

The results presented were calculated considering the stress range spectrum shown in Fig. 1. A cutoff limit of $9 \mathrm{MPa}$ was set to eliminate disturbances from the measurement equipment. The mean value is $23,3 \mathrm{MPa}$ and the standard deviation is $6,71 \mathrm{MPa}$.

The expected crack growth is shown in Fig. 3 for two cases. First, a threshold on the stress intensity range $K_{\text {th }}$ was considered as stated in Table 1 which gives an expected fatigue life of about 290 million cycles. Secondly, the threshold was omitted which imply that all stress ranges contribute to the crack propagation giving an expected fatigue life of about 34 million cycles. It is evident that the threshold has a paramount influence on the fatigue life for the treated detail and given stress range spectrum.

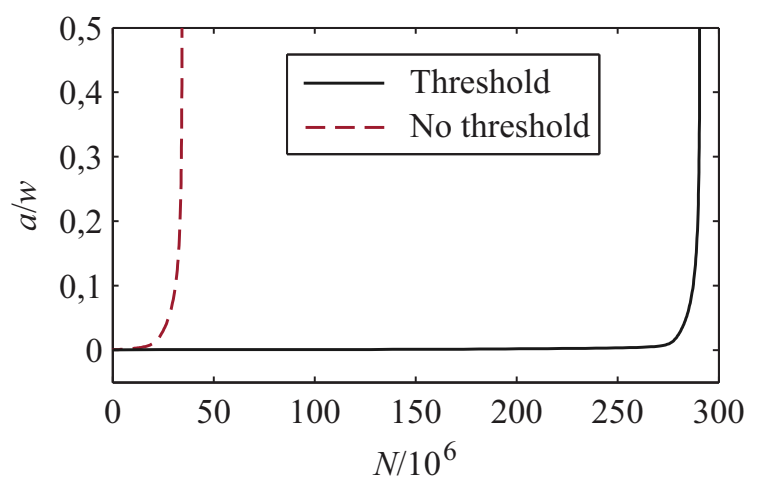

Figure 3. The expected crack growth for the reference case with and without a threshold value on the stress intensity factor range.

It is evident in Fig. 3 that the crack depth is very small over a considerable part of the fatigue life. It should, however, be noted that this result is valid for this specific detail and stress range spectrum.

\subsection{Fatigue crack growth threshold}

The presence of a threshold on the stress intensity factor range is well established and supported by guidelines such as [7] and [13]. For welded components in air or other non-aggressive environments with temperatures up to $100^{\circ} \mathrm{C}$ a deterministic value of $63 \mathrm{~N} / \mathrm{mm}^{3 / 2}$ is suggested. This conservative value represents the $3 \%$-fractile of the distribution stated in Table 1.

The importance of the threshold value is already indicated in Fig. 3. The influence on the estimated fatigue life based on reliability is shown in Fig. 4. For a target reliability of $\beta=3,1$ the fatigue life is 16 and 6,5 million cycles with and without any threshold, respectively.

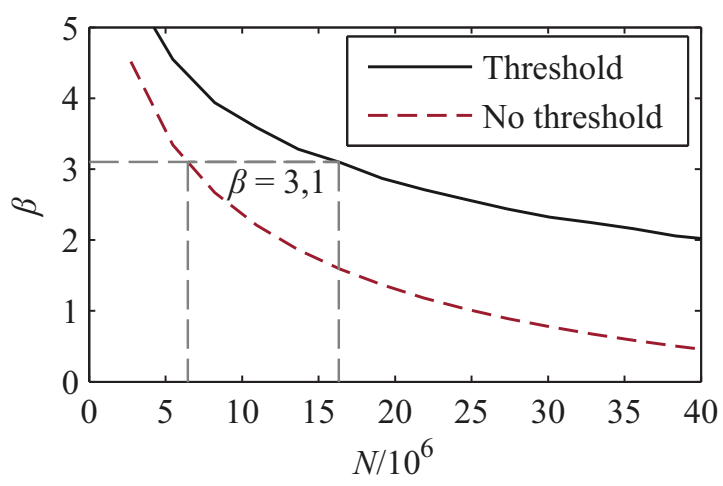

Figure 4.The accumulated reliability index showing the influence of the crack growth threshold.

\subsection{Material dependent properties}

The bi-linear crack growth rate described by Eq. (7) and its material parameters stated in Table 1 originates from a review of crack propagation tests of offshore steels presented in [14]. The bilinear relation is shown to be more accurate than the linear Paris law. If a linear relation is preferred, the variable $A$ is suggested to be lognormal distributed with a mean value of $2,5 \cdot 10^{-13}$ and a CoV of 0,54 . The exponent $n$ is suggested as deterministic with a value of 3 .

The textbook [15] specifically treats the fatigue assessment of steel bridges. There, a linear relation is suggested with the variable $A$ as lognormal distributed with a mean value of $1,24 \cdot 10^{-13}$ and a CoV of 0,63 . The exponent $n$ is 
suggested as deterministic with a value of 3 . The origin of these values is unclear.

The paper [16] is a rare example presenting crack growth data for specimens from existing bridges. These data representing three tests from bridges in Sweden are too few to provide any base for a statistical evaluation but comparisons with the distributions mentioned before are shown in Fig. 5. The statistical distributions are shown as $95 \%$ confidence intervals. The result from the three tests lies within the confidence intervals for both suggested crack growth rates.

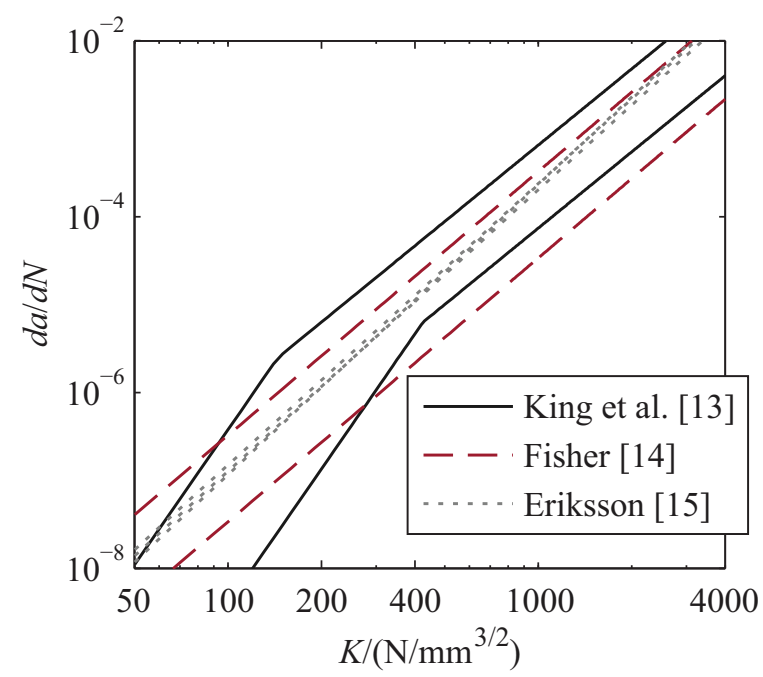

Figure 5. Fatigue crack growth rates from different sources.

The influence of different crack growth rates on the estimated fatigue life is shown in Fig. 6 . The difference between a linear and a bi-linear crack growth rate is apparent but not significant. Somewhat surprisingly, the linear crack growth rate gives a longer fatigue life than the bi-linear for the current case. It depends on the portion of stress intensity ranges below or above the knee point of the bi-linear function. For a target reliability of $\beta=3,1$ the fatigue life is 16 and 19 million cycles for a bi-linear and a linear growth rate, respectively.

The crack growth rate suggested by Fisher [15] gives a significantly longer estimated fatigue life than the bi-linear and linear rates, both suggested by King et al. [14]. As indicated in Fig. 6, a fatigue life of 34 million cycles was reached for the rate suggested in [15].

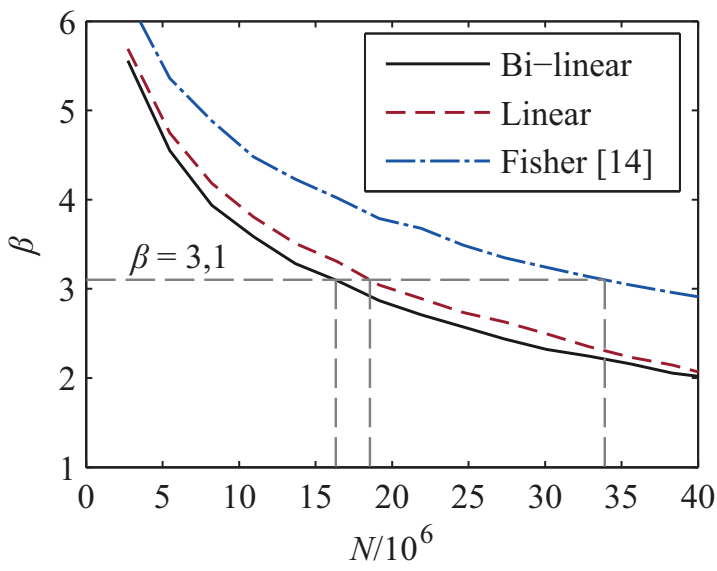

Figure 6. The accumulated reliability index showing the influence of different crack growth rates.

\subsection{Omission sensitivity factors}

The sensitivity of the reliability to the uncertainties of the stochastic variables can be evaluated using the so-called omission sensitivity factors [17]. These are defined as the ratio between the reliability index with one of the variables replaced by a deterministic value $x_{i}^{\mathrm{f}}$ and the original reliability index. The factors are calculated as [17]

$$
\gamma_{i}=\frac{\beta\left(x_{i}=x_{i}^{\mathrm{f}}\right)}{\beta}=\frac{\beta-\alpha_{i} u_{i}^{\mathrm{f}}}{\beta \sqrt{1-\alpha_{i}^{2}}}
$$

where $\alpha_{i}$ is the direction cosine for variable $i$ calculated within the FORM at the design point. The variable $u_{i}^{\mathrm{f}}$ is the normalized coordinates of the deterministic value $x_{i}^{\mathrm{f}}$.

The omission sensitivity factors calculated for the reference model with the stochastic variables in Table 1 are shown in Fig. 7. The calculation was made for a total of 16 million cycles distributed as shown in Fig. 1. The deterministic values $x_{i}^{\mathrm{f}}$ were set equal to the mean values of the variables. An omission factor close to unity indicates that the stochastic variable can be replaced by a deterministic value. 


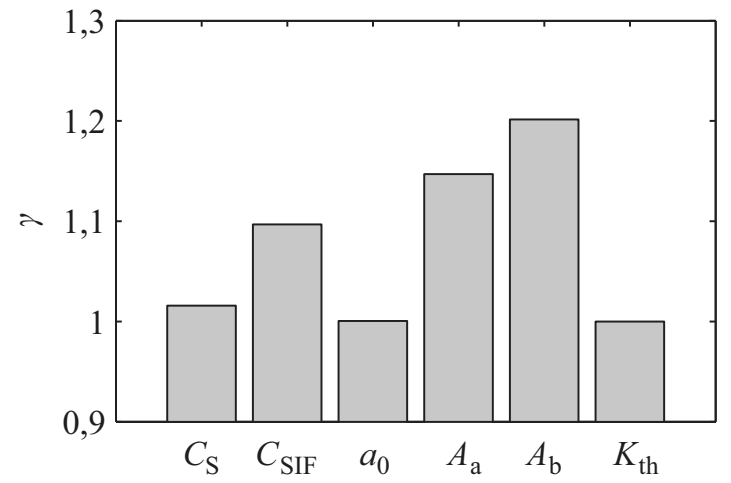

Figure 7. Omission sensitivity factors calculated for the reference model at 16 million cycles.

It is evident from Fig. 7 that the uncertainties of the material parameters $A_{\mathrm{a}}$ and $A_{\mathrm{b}}$ have an important influence on the estimated reliability. So has the uncertainty of the stress intensity factor $C_{\mathrm{SIF}}$. The uncertainties of the initial crack depth and the crack growth threshold do not have any significant influence on the reliability. These variables have, however, a significant influence on the estimated fatigue life which for the threshold value is shown in Fig. 4.

\section{Conclusions}

A probabilistic model is suggested for fatigue life prediction using linear elastic fracture mechanics (LEFM). This model is used for a sensitivity analysis on different modelling choices for a specific connection and a stress range spectrum based on measured response. The conclusions are valid for this specific case but the study gives indications on important parameters in general. Considering the result, the following conclusions can be drawn:

- The crack growth threshold can have a significant influence on the fatigue life if the detail and the remote stress together cause low stress intensity factor ranges. The fatigue life with and without the threshold value differs with a factor of 2,5 for the studied case.

- The difference in fatigue life between a linear and a bi-linear crack growth rate is apparent but small. For the studied case, the difference is less than $20 \%$.

- The crack growth rate suggested by Fisher [15] for steel bridges gives a significantly longer fatigue life than what is obtained with the suggestion in King et al. [14] reproduced in BS 7910. The difference is a factor 2,1 for the studied case.

- The omission sensitivity factors indicate the uncertainties related to the material parameters and the stress intensity factor as important for the estimated reliability.

- The uncertainties of the initial crack depth and the crack growth threshold have only a modest influence on the reliability. This means that these variables could be considered as deterministic in the assessment.

\section{Acknowledgements}

The funding for this project provided by the Swedish Transport Administration (Trafikverket), BBT project number 2013-004, is gratefully acknowledged.

\section{References}

[1] Leander J, Andersson A, Karoumi R. Monitoring and enhanced fatigue evaluation of a steel railway bridge. Engineering Structures. 2010; 32(3): 854863.

[2] Al-Emrani M, Kliger R. Fatigue prone details in steel bridges. Nordic Steel Construction Conference. Malmö, Sweden; 2009.

[3] Broek D. The practical use of fracture mechanics. Dordrecht, the Netherlands: Kluwer Academic Publishers; 1998.

[4] Paris PC, Gomez MP, Andersson WE. A rational analytic theory of fatigue. The Trend in Engineering. 1961; 13(1): 9-14.

[5] Hobbacher AF. Stress intensity factors of plates under tensile load with welded-on flat side gussets. Engineering Fracture Mechanics. 1992; 41(6): 897-905.

[6] Melchers RE. Structural reliability analysis and prediction. West Sussex: John Wiley \& Sons, Inc.; 2002. 
[7] BS 7910. Guide to methods for assessing the acceptability of flaws in metallic structures. British Standards (BSi); 2007.

[8] JCSS. Probabilistic model code - Part 3: Resistance models. Joint Committee on Structural Safety (JCSS); 2011.

[9] Matlab [computer program]. Version R2011a. The MathWorks, Inc. Natick, Massachusetts, US; 2011.

[10] Leander J, Norlin B, Karoumi R. Reliabilitybased calibration of fatigue safety factors for existing steel bridges. ASCE Journal of Bridge Engineerin. 2015; 20(10).

[11] Tada H, Paris PC, Irwin GR. The stress analysis of cracks handbook. New York, US: ASME Press; 2000.

[12] Leander J, Aygül M, Norlin B. Refined fatigue assessment of joints with welded inplane attachments by LEFM. International Journal of Fatigue. 2013; 56.

[13] Hobbacher AF. Recommendations for fatigue design of welded joints and components, IIW document XIII-215107/XV-1254-07. International Institute of Welding; 2007.

[14] King RN, Stacey A, Sharp JV. A review of fatigue crack growth rates for offshore steels in air and seawater environments. Proceedings of the $15^{\text {th }}$ International Conference on Offshore Mechanical and Artic Engineering. OMAE-96, Vol. III, ASME; 1996.

[15] Fisher JW. Fatigue and fracture in steel bridges - Case studies. New York, US: John Wiley \& Sons, Inc.; 1984.

[16] Eriksson K. Toughness requirements for older structural steels. IABSE Symposium Report. Lausanne; 1990.

[17] Madsen HO. Omission sensitivity factors. Structural Safety. 1998; 5(1): 35-45. 